\title{
Direct conversion of astrocytes into neuronal cells by drug cocktail
}

Cell Research (2015) 25:1269-1272. doi:10.1038/cr.2015.120; published online 2 October 2015

\section{Dear Editor,}

Neurological disorder is one of the greatest threats to public health according to the World Health Organization. Because neurons have little or no regenerative capacity, conventional therapies for neurological disorders yielded poor outcomes. While the introduction of exogenous neural stem cells or neurons holds promise, many challenges still need to be tackled, including cell resource, delivery strategy, cell integration and cell maturation. Reprogramming of fibroblasts into induced pluripotent stem cells or directly into desirable neuronal cells by transcription factors (TFs) or small molecules can solve some problems, but other issues remain to be addressed, including safety, conversion efficiency and epigenetic memory $[1,2]$.

Astrocytes are considered to be the ideal starting candidate cell type for generating new neurons, due to their proximity in lineage distance to neurons and ability to proliferate after brain damage. Many studies have already revealed that astrocytes of the central nervous system can be reprogrammed into induced neuronal cells by virus-mediated overexpression of specific TFs in vitro and in vivo [3-6]. However, application of this virus-mediated direct conversion is still limited due to concerns on clinical safety. We have previously reported direct conversion of somatic cells into neural progenitor cells (NPCs) in vitro by cocktail of small molecules under hypoxia [7]. Here we set out to explore whether astrocytes can be induced into neuronal cells by the chemical cocktail in vitro.

We first isolated astrocytes from 1-day postnatal mouse brain. To exclude contamination of neuronal cells and NPCs in primary astrocytes, we strictly followed the isolation protocol and used cells passaged at least three times in each experiment. Moreover, retrovirus-mediated delivery of GFAP::GFP reporters into these cells, which selectively label proliferative astrocytes, but not neuronal cells, further confirmed the purity of astrocytes (Supplementary information, Figure S1A-S1D) [3]. Then we treated cultured astrocytes with the chemical cocktail
VCR (V, VPA, 3 mM; C, CHIR99021, 3 M; R, Repsox, $1 \mu \mathrm{M})$. The same cocktail reported in our previous study [7] was used here with slight modification, such as the optimized VPA concentration. Briefly, astrocytes cultured in astrocyte medium for $48 \mathrm{~h}$ were transferred into DMEM/F12 medium that contains the VCR cocktail and other supplements including B27, N2, $20 \mathrm{ng} / \mathrm{ml}$ BDNF and $20 \mathrm{ng} / \mathrm{ml}$ GDNF. The VCR-containing medium was changed every 4 days. After 8 days under VCR treatment, cells were maintained in DMEM/F12 medium (containing $400 \mathrm{ng} / \mathrm{ml} \mathrm{shh}, 100 \mathrm{ng} / \mathrm{ml} \mathrm{FGF8,} 10 \mathrm{ng} / \mathrm{ml}$ bFGF, $20 \mu \mathrm{M}$ L-Ascorbic acid, $20 \mathrm{ng} / \mathrm{ml}$ BDNF, $20 \mathrm{ng} /$ $\mathrm{ml} \mathrm{GDNF}, \mathrm{B} 27$ and N2) without the cocktail under hypoxia. Astrocytes were added to fill the blank and support the development of induced neuronal cells when necessary. Obviously, VCR treatment changed the cell morphology from astrocyte- into neuronal cell-like morphology, as shown by the phase contrast images (Figure 1A). Importantly, cultured mouse astrocytes can be induced into $\mathrm{DCX}^{+}$neuroblasts (Figure 1B) and Tuj $1^{+}$or $\mathrm{NeuN}^{+}$ neurons (Figure 1C) 12 days and 18 days post induction, respectively. Quantification of conversion efficiency showed that $\mathrm{DCX}^{+}$cells appeared as early as 3 days post induction, and the number of $\mathrm{DCX}^{+}$cells peaked on day 12 and then slightly decreased. In contrast, $\mathrm{NeuN}^{+}$cells were detectable from day 6 and their number gradually increased afterwards (Supplementary information, Figure $\mathrm{S} 1 \mathrm{E})$. The dynamic alteration in expression of neuronal cell markers in a continuous process reinforces the idea that induced neuronal cells are generated from starting astrocytes. Importantly, we observed that mature neurons induced from cultured astrocytes fired repetitive trains of action potentials and exhibited postsynaptic current spontaneously (Figure 1D-1F). During the whole conversion process from astrocytes to neuronal cells, we did not observe emergence of Sox $2^{+}$NPCs (Supplementary information, Figure S2A and S2B). Moreover, NPCs were also absent even when astrocytes were cultured under the same condition used for NPC induction from fibroblasts [7].

To tease out critical constituent(s) enabling astrocyt- 
A

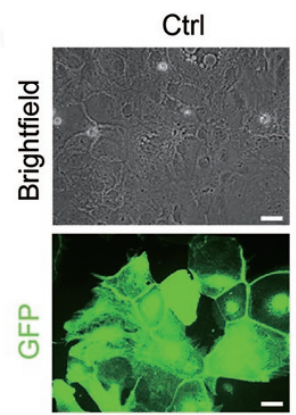

C

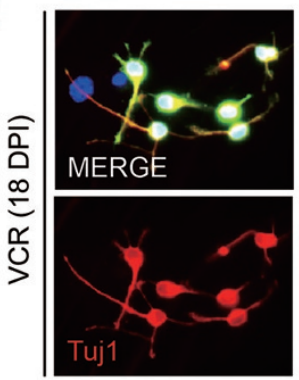

D

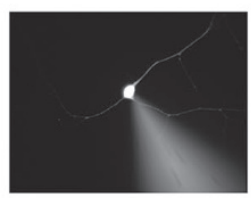

G

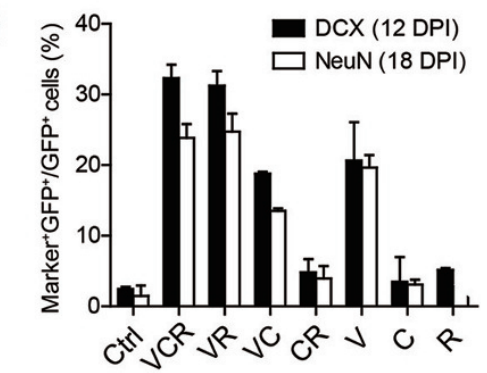

VCR
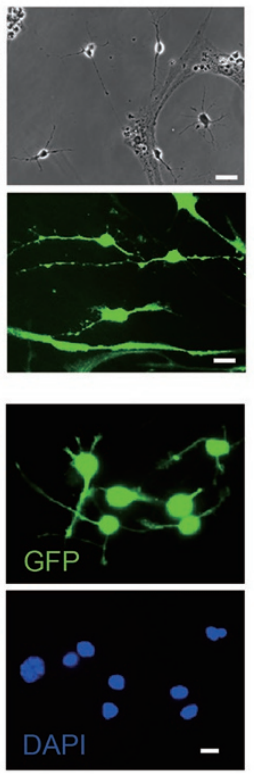

B
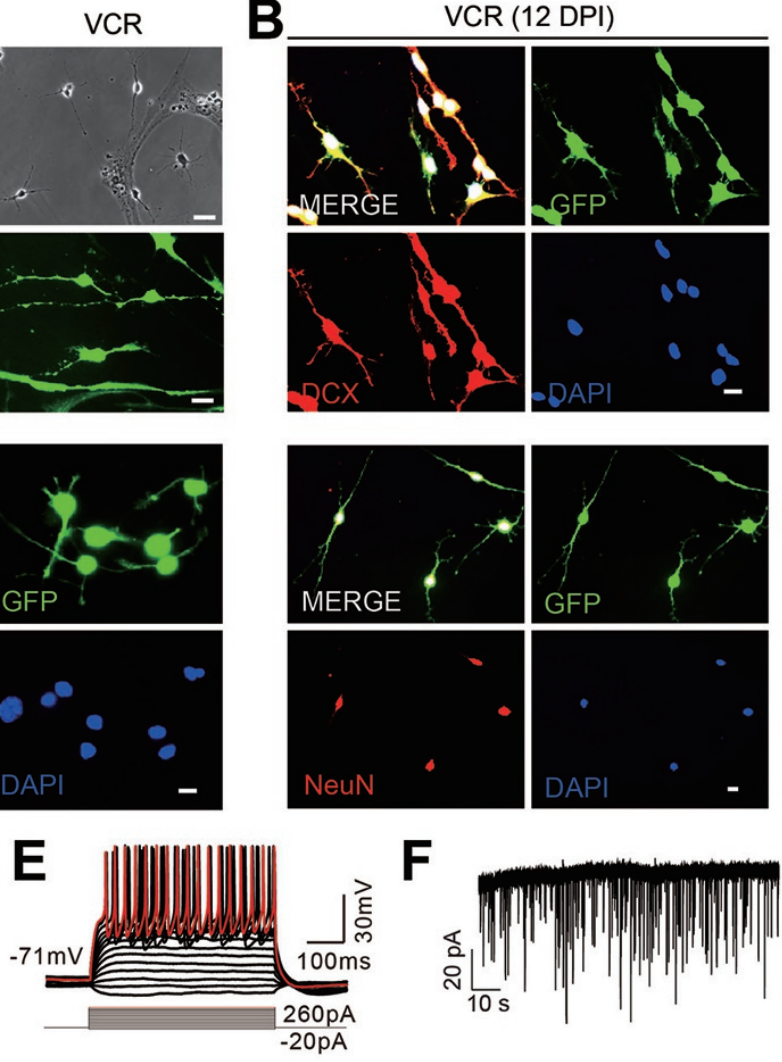

H
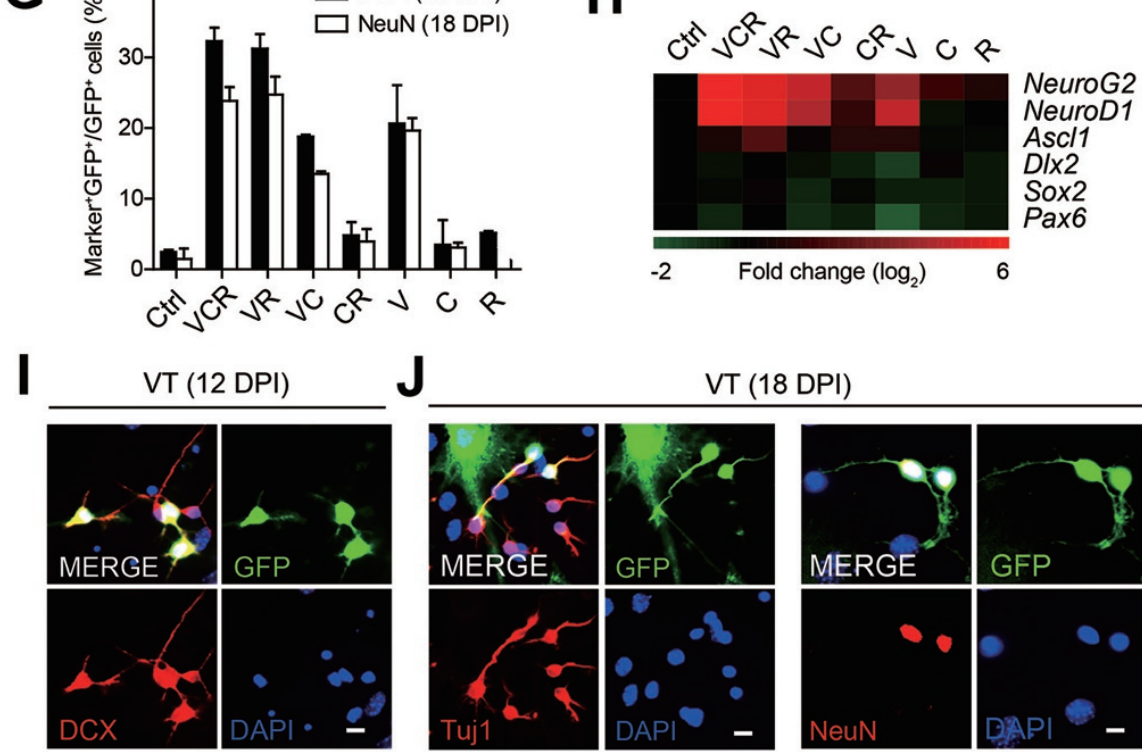

J

VT (18 DPI)
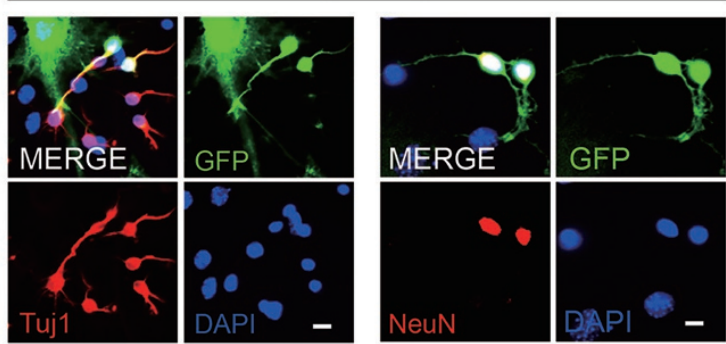

Figure 1 Cultured astrocytes are induced into neuronal cells by small molecules. (A) Cultured astrocytes showed neuronal morphology 12 days post VCR induction (DPI, days post induction). GFP ${ }^{+}$cells stand for astrocytes labelled by GFAP::GFP. (B) Cultured astrocytes were converted into $\mathrm{DCX}^{+}$neuoroblasts 12 days post VCR induction. (C) Neuronal cells converted from astrocytes expressed mature neuron markers Tuj1 and NeuN 18 days post VCR treatment. (D) Patch-clamp recording was conducted on VCR-induced neurons from cultured astrocytes identified by fluorescence 26 days post induction. (E) Current-clamp recordings of neurons derived from cultured astrocytes with VCR treatment showed a representative train of action potentials with stepwise current injection. (F) Representative traces of spontaneous postsynaptic currents recorded in VCR-induced neurons from astrocytes. (G) Proportion of GFP ${ }^{+}$cells co-expressing DCX or NeuN in the final GFP ${ }^{+}$cells. (H) Heat map depicting the relative fold changes of gene expression levels in astrocytes 1 week after chemical treatment in vitro. The value in the color bar indicates log2 changes (relative to HPRT and normalized to ctrl). (I, J) DCX+, Tuj $1^{+}$and $\mathrm{NeuN}^{+}$cells were generated from cultured astrocytes under treatment of the drug cocktail VT. Data are presented as mean \pm SEM; scale bar, $10 \mu \mathrm{m}$. 
ic-to-neuronal transition within the chemical cocktail, we conducted screens by removing one or two component(s) from the cocktail. Elimination of VPA significantly abolished the generation of neuroblasts from astrocytes, whereas the exclusion of CHIR99021 or Repsox had minimal effect on neuroblast induction under our culturing conditions. Interestingly, conversion induced by VR (VPA and Repsox) was as efficient as that by the VCR cocktail. In contrast, VPA alone was able to induce astrocytes into neuroblasts with lower efficiency compared with VR, while neither CHIR99021 or Repsox alone nor their combination produced any induced neuroblasts (Supplementary information, Figure S1F and S1G). Later these chemical-induced neuroblasts developed into mature neurons expressing NeuN with diverse efficiencies (Supplementary information, Figure S1H). Calculation of $\mathrm{DCX}^{+}$or $\mathrm{NeuN}^{+}$cell proportion in the final population (Figure 1G) or conversion efficiency (neuronal marker-positive cell number/initial cell number; Supplementary information, Figure S1I) at different time points clearly demonstrates that the critical chemical components in VCR are VPA and Repsox. Mature neurons of specific types were also generated from astrocytes 25 days post VR induction, including dopaminergic neurons, GABAergic neurons, glutamatergic neurons and motor neurons (Supplementary information, Figure S1J-S1N). The glycogen synthase kinase 3 inhibitor CHIR99021 has been reported to strongly enhance NeuroD1 expression in NPCs, which leads to neuronal differentiation from NPCs [8]. However, CHIR99021 did not activate NeuroD1 expression or facilitate neuronal conversion in our system, which also excludes the possibility of contamination and generation of NPCs here. In addition, we did not find any chemical component in VCR or their combination that contributed to proliferation of the starting cells or neurons, further excluding the possibility of neuron contamination in starting cells (Supplementary information, Figure S2C-S2E).

To analyze the potential underling mechanisms, we first checked the expression of TFs including NeuroG2, NeuroD1, Ascl1, Pax6, Dlx2 and Sox2, each of which has been reported to sufficiently reprogram astrocytes into neuronal cells [9]. Surprisingly, no chemical or chemical cocktail was able to activate Ascl1, Dlx2, Sox2 and Pax6 expression. Cocktails containing VPA or even VPA alone could significantly activate the expression of NeuroG2 and NeuroD1 (Figure 1H). Among these two TFs, NeuroD1-induced astrocytic-to-neural conversion was also confirmed in our system (Supplementary information, Figure S2F and S2G). These two TFs contain the basic helix-loop-helix (bHLH) DNA-binding domain. Since Notch signaling regulates bHLH factors as a con- served module and also regulates neurogenic program of astrocytes after brain stroke [10], we next investigated the expression pattern of genes involved in this pathway. However, treatment with the chemical(s) did not cause any significant change in the expression of Notch receptors $1 / 2 / 3 / 4$ and Notch ligands Dll1/3/4, Jagged 1/2 and Hes 1/5 (Supplementary information, Figure S2H and Table S1). Based on these findings, we suggest that chemicals capable of inducing astrocytic-to-neuronal transition might act by inducing NeuroG2 and NeuroD1 expression independently of Notch signaling.

Our previous study showed that components of chemical cocktails capable of inducing cell reprogramming can be substituted with other small molecules targeting the same signaling pathway [7]. Thus, we wonder whether it is possible to replace chemicals in the cocktail with pharmaceutical compounds, which might be directly applied in vivo for translational medicine. The reduced-chemical cocktail VR contains VPA and Repsox that can inhibit histone deacetylases and TGF- $\beta$ signaling, respectively. We kept VPA (an anticonvulsant and mood-stabilizing drug), replaced Repsox by another TGF- $\beta$ inhibitor Tranilast ( $\mathrm{T}$, an antiallergic drug, $100 \mu \mathrm{M}$ ), and then tested the neuronal induction capacity of this new drug cocktail (termed as VT). As expected, $\mathrm{DCX}^{+}$cells were generated from cultured astrocytes 12 days post induction using VT (Figure 1I). Neurons expressing Tuj1 or NeuN were also observed 18 days post induction (Figure $1 \mathrm{~J}$ ). Consistently, VT also significantly activated expression of NeuroG2 and NeuroD1 in astrocytes under the conversion conditions (Supplementary information, Figure S2I).

In summary, our results demonstrate that small molecules can directly convert astrocytes into neurons in vitro through activation of NeuroG2 and NeuroD1 expression. Using small molecules to manipulate this cell fate transition can be an attractive approach for clinical application. Importantly, we also found that the chemical cocktail VCR can enable astrocytes of adult mice to acquire neuronal cell properties in vitro (Supplementary information, Figure S2J-S2L), suggesting the practical implication of such chemical-induced conversion in vivo. The potential effects of these chemicals on other types of cells, such as neurons, need to be systematically and carefully evaluated in the future. Nevertheless, local delivery of small molecules or systematic administration of drug cocktail enabling astrocytic-to-neuronal conversion might achieve direct induction of desirable cells from resident astrocytes in situ to meet the ultimate goal of regenerative medicine.

\section{Acknowledgments}


We thank all members of the lab for sharing reagents and advice. This work was supported by the Chinese Academy of Sciences (XDA01010302), the Ministry of Science and Technology (2015CB964502, 2013CB966801 and 2011CB946102), the Ministry of Health (2012BAI10B03), the National Natural Science Foundation of China (31301129), the Shanghai Municipal Commission for Science and Technology (12ZR1452300), and the Samuel Waxman Cancer Research Foundation Co-PI Program.

\section{Lin Cheng ${ }^{1,4,}$, Longfei Gao ${ }^{1, *}$, Wuqiang Guan ${ }^{2}$, Jianxin Mao ${ }^{1}$, Wenxiang $\mathrm{Hu}^{1}$, Binlong Qiu ${ }^{1}$, Jian Zhao ${ }^{1}$, Yongchun $\mathrm{Yu}^{2}$, Gang Pei ${ }^{1,3}$}

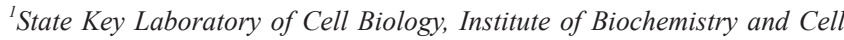
Biology, Shanghai Institutes for Biological Sciences, Chinese Academy of Sciences, Shanghai 200031, China; ${ }^{2}$ State Key Laboratory of Medical Neurobiology, Shanghai Medical College and Institutes of Brain Science, Fudan University, Shanghai 200032, China; ${ }^{3}$ Collaborative Innovation Center for Brain Science, School of Life Science and Technology, Tongji University, Shanghai 200092, China; ${ }^{4}$ State Key Laboratory of Medical Genomics, Shanghai Institute of Hematology, Rui Jin Hospital, Shanghai Jiao Tong University School of Medicine, Shanghai 200025, China

*These two authors contributed equally to this work.

Correspondence: Gang Pei

E-mail: gpei@sibs.ac.cn

\section{References}

1 Hu W, Qiu B, Guan W, et al. Cell Stem Cell 2015; 17:204-212.

2 Li X, Zuo X, Jing J, et al. Cell Stem Cell 2015; 17:195-203.

3 Guo Z, Zhang L, Wu Z, et al. Cell Stem Cell 2014; 14:188-202.

4 Niu W, Zang T, Zou Y, et al. Nat Cell Biol 2013; 15:1164-1175.

5 Liu ML, Zang T, Zou Y, et al. Nat Commun 2013; 4:2183.

6 Heinrich C, Blum R, Gascon S, et al. PLoS Biol 2010; 8:e1000373.

7 Cheng L, Hu W, Qiu B, et al. Cell Res 2014; 24:665-679.

8 Kuwabara T, Hsieh J, Muotri A, et al. Nat Neurosci 2009; 12:10971105.

9 Amamoto R, Arlotta P. Science 2014; 343:1239882.

10 Magnusson JP, Goritz C, Tatarishvili J, et al. Science 2014; 346:237241

(Supplementary information is linked to the online version of the paper on the Cell Research website.)

(c) (i) () $€$ This work is licensed under a Creative Commons Attribution-NonCommercial-NoDerivs 4.0 Unported License. The images or other third party material in this article are included in the article's Creative Commons license, unless indicated otherwise in the credit line; if thematerial is not included under the Creative Commons license, users will need to obtain permission from the license holder to reproduce the material. To view a copy of this license, visit http://creativecommons.org/licenses/by-nc-nd/4.0/ 